\title{
Benefícios do banho tardio no recém-nascido: implicações para a enfermagem
}

\author{
Benefits of the delayed bath in the newborns: implications for nursing \\ Beneficios del baño tardío en el recién nacido: implicaciones para la enfermería \\ Tuane Scarabonatti Cantoni ${ }^{1 *}$, Rossano Sartori Dal Molin¹.
}

\begin{abstract}
RESUMO
Objetivo: Identificar os benefícios do banho tardio no recém-nascido e suas implicações na prática da enfermagem. Métodos: Trata-se de um estudo do tipo revisão integrativa de literatura, a coleta dos dados foi realizada nas bases de dados do sítio da Biblioteca Virtual em Saúde (BVS) - BIREME: Medical Literature Analysis and Retrieval System Online (MEDLINE) e Scientific Eletronic Library Online (SCIELO) através dos descritores: recém-nascido e banho, sendo incluídos apenas artigos dos últimos cinco anos. Resultados: No total foram identificados 783 artigos, sendo elencados como relevantes para serem analisados na presente revisão seis. Somente dois estudos avaliaram o banho tardio considerando no mínimo 24 horas de vida do $\mathrm{RN}$ e ambos obtiveram resultados positivos relacionados a termorregulação. O banho tardio está relacionado a prevenção da hipotermia neonatal, não remoção precoce do vérnix, promoção do contato pele a pele, vínculo materno e principalmente na inserção dos pais nos cuidados com o recém-nascido. Considerações finais: $O$ banho tardio preferencialmente após às 24 horas de vida, está diretamente relacionado a uma melhor adaptação do recém-nascido ao ambiente extrauterino e vínculo materno.
\end{abstract}

Palavras-chave: Recém-nascido, Banho, Enfermagem neonatal.

\begin{abstract}
Objective: Identify the benefits of the practice of delaying bathing in newborns and its implications for nursing practice. Methods: This is an integrative literature review study, data collection took place in the databases of the Virtual Health Library (VHL) website - BIREME: Medical Literature Analysis and Retrieval System Online (MEDLINE) and Scientific Eletronic Library Online (SCIELO) through the descriptors: newborn and bath, including only articles from the last five years. Results: In total, 783 articles were identified, 6 being listed as relevant to be analyzed in the present review. Only two studies evaluated the delayed bath considering at least 24 hours of life for the newborn and both obtained positive results related to thermoregulation. Delaying bathing is related to the prevention of neonatal hypothermia, delayed vernix removal, promotion of skin contact, maternal bonding and especially the insertion of parents in the care of the newborn. Final considerations: The delayed bath, preferably after 24 hours of life, is directly related to a better adaptation of the newborn to the extrauterine environment and maternal bond.
\end{abstract}

Keywords: Newborn, Bath, Neonatal nursing.

\section{RESUMEN}

Objetivo: Identificar los beneficios de la práctica del baño tardío en el recién nacido y sus implicaciones para la práctica de enfermería. Métodos: Se trata de un estudio integrador de revisión de la literatura, la recolección de datos se realizó en las bases de datos del sitio web de la Biblioteca Virtual en Salud (BVS) - BIREME:

${ }^{1}$ FSG Centro Universitário, Caxias do Sul - RS. *E-mail: tuane_539@hotmail.com 
Sistema de análisis y recuperación de literatura médica en línea (MEDLINE) y Scientific Eletronic Library Online (SCIELO) a través de los descriptores: recién nacido y baño, incluyendo solo artículos de los últimos cinco años. Resultados: En total, se identificaron 783 artículos, siendo catalogados como relevantes para ser analizados en la presente revisión 6 . Solo dos estudios evaluaron el baño tardío considerando al menos 24 horas de vida para el recién nacido y ambos obtuvieron resultados positivos relacionados con la termorregulación. El baño tardío está relacionado con la prevención de la hipotermia neonatal, no con la remoción temprana del vérnix, la promoción del contacto con la piel, el vínculo materno y especialmente la inserción de los padres en el cuidado del recién nacido. Consideraciones finales: El baño tardío, preferentemente a las 24 horas de vida, está directamente relacionado con una mejor adaptación del recién nacido al ambiente extrauterino y vínculo materno.

Palabras clave: Recién nacido, Baño, Enfermería neonatal.

\section{INTRODUÇÃO}

A evolução socioeconômica somada ao aprimoramento técnico-científico, modificou a forma de nascimento na atualidade, fazendo com que as mulheres passassem a ser assistidas no momento do parto por equipes médicas em Instituições Hospitalares. Com isso, visando minimizar riscos de agravos a saúde do bebê e da mãe, surgiram os berçários centralizados e salas de recuperação pós parto, desencadeando na separação do binômio mãe-bebê. No entanto, diante de pesquisas relacionadas ao prejuízo do vínculo materno, criou-se o Sistema Alojamento Conjunto que favorece a interação e a participação ativa dos pais nos cuidados ao recém-nascido, aproximando-se, do que ocorria há algumas décadas: o nascimento em ambiente domiciliar, que possibilita o acompanhamento do processo por pessoas conhecidas pela mulher, a qual possui confiança e após, permanência na presença da família, proporcionado um momento oportuno para criação de vínculo (CARVALHÊDO SD, et al., 2010).

A adaptação fisiológica do recém-nascido $(\mathrm{RN})$ à vida extrauterina acontece nas primeiras 24 horas de vida e torna-se completa quando os sinais vitais, sua capacidade de alimentação e as funções gastrointestinais e renais estão estabilizadas. Devido à importância deste período, a recomendação da prática do primeiro banho necessita ser baseada em evidências para que não interfira no período da adaptação fisiológica. Neste sentido, a Organização Mundial de Saúde (OMS) recomenda que a prática do primeiro banho seja realizada após as primeiras 24 horas de vida, embasando-se nos benefícios que o vérnix caseoso proporciona para o recém-nascido, facilitando a adaptação deste ao ambiente extrauterino. (CUNHA AL, 2013; RUSCHEL LM, et al., 2018; WHO, 2017).

O banho do RN é um procedimento rotineiro nas unidades neonatais, porém questiona-se cada vez mais quais são os efeitos deste no processo de transição do meio intrauterino para o meio extrauterino e qual seria o momento ideal para a sua realização. A manutenção da temperatura corpórea é um dos fatores determinantes para o sucesso da estabilização cardiocirculatória e respiratória do RN imediatamente após o nascimento e o banho implica diretamente nesse fator. As complicações que mais se associam ao aumento da morbidade e mortalidade dos recém-nascidos são a dificuldade em manter a termorregulação e a síndrome do desconforto respiratório (FREITAS P, et al., 2014; PUGLIESI VEM, et al, 2009; RUSCHEL LM, et al., 2018).

Realizar o banho no recém-nascido impacta em uma série de estímulos. Os recém-nascidos podem responder através da mudança do seu estado comportamental aos estímulos externos ou internos, estes estados refletem a organização interna do bebê e sua capacidade de controlar os estímulos externos. Assim, a prática do primeiro banho pode alterar os sinais vitais e a sua proteção térmica, que quando modificada pode causar hipotermia, aumentando o consumo de oxigênio e consequentemente aumento da frequência respiratória, acarretando em um estresse. Sendo assim, o banho deve ser realizado de forma adequada, sendo o de imersão o mais indicado, utilizando-se de água morna, visto permitir menor perda de calor e conforto pro bebê, além disso, ele deve ser realizado por profissional capacitado que vise um cuidado integral, com tranquilidade e segurança (CARVALHÊDO SD, et al., 2010; LIMA RO, et al., 2020). 
O papel da enfermagem também está atrelado a educação em saúde. O ambiente do Alojamento Conjunto é de suma importância, visto possibilitar que a família desenvolva os cuidados com o recém-nascido sob orientações, supervisões e demonstrações do enfermeiro, e entre estes cuidados a técnica do banho, a qual representa a maior vulnerabilidade da família, sendo preciso demonstrá-la individualmente para cada familiar. Além disso, possibilita que a equipe oriente sobre as técnicas de higienização do coto umbilical e auxilie na amamentação (CARVALHÊDO SD, et al., 2010; LIMA RO, et al., 2020).

No Brasil atualmente, a mortalidade neonatal é responsável por quase $70 \%$ das mortes no primeiro ano de vida, sendo que $25 \%$ representam mortes na primeira semana de vida. Historicamente, o primeiro banho do RN tem práticas culturais fortemente associadas a ele, seja social, familiar ou profissional, que permanecem até hoje impactando na assistência. O cuidado adequado ao neonato tem sido um dos principais desafios para reduzir os índices de mortalidade infantil (BRASIL, 2014; FREITAS P, et al., 2014; PRIORE MD, 2010).

O presente estudo justificou-se pela relevância do tema, considerando a extrema importância de pesquisar a respeito dos primeiros cuidados prestados ao recém-nascido e o impacto destes na adaptação extrauterina. Desse modo, propõe-se com esta revisão, identificar os benefícios do banho tardio no recém-nascido, bem como, se há um momento ideal para a realização do mesmo e o impacto da assistência de enfermagem neste processo.

\section{MÉTODOS}

Trata-se de um estudo do tipo revisão integrativa de literatura, visto ser um método de pesquisa utilizado na prática baseada em evidências, já que a análise de pesquisas relevantes dá suporte para a tomada de decisão, fundamentação de condutas e proporciona um saber crítico que resulta em melhoria da prática clínica. Para isso, utilizou-se um processo de pesquisa que incluiu cinco etapas: formulação do problema, coleta de dados, avaliação dos dados, análise e interpretação dos dados, apresentação dos resultados (COOPER HM, 1982; MENDES KDS, et al., 2008).

Para o procedimento de seleção dos artigos o estudo foi norteado pela seguinte questão: "Quais são os benefícios do banho tardio no recém-nascido?". A coleta de dados foi realizada em duas importantes bases de dados do sítio da Biblioteca Virtual em Saúde (BVS) - BIREME: MEDLINE (Literatura Internacional em Ciência da Saúde) e SCIELO (Scientific Eletronic Library Online). Os Descritores em Ciências da Saúde (DeCS) para a busca dos artigos foram: banho e recém-nascido.

Os estudos incluídos na presente revisão integrativa obedeceram aos seguintes critérios de inclusão: artigos que abordassem a temática e o objetivo do estudo, artigos originais, disponíveis online, na íntegra, escritos nos idiomas português, inglês e espanhol e publicados de 2015 a 2020. Foram excluídos da pesquisa artigos que não estavam na integra, que não responderam ao objetivo do estudo e artigos de revisão.

A avaliação dos dados se deu por meio da leitura criteriosa dos artigos, utilizando-se do julgamento crítico, extraindo informações e as registrando em um instrumento de coleta previamente elaborado contento os seguintes itens: Dados de identificação do artigo, objetivo/questão norteadora, método, resultados, conclusões e observações. Esta metodologia facilitou a identificação dos estudos mais pertinentes e que estavam realmente relacionados com a questão norteadora.

$\mathrm{Na}$ etapa de análise e interpretação dos dados, as informações foram articuladas e sintetizadas e representadas através de um quadro, exibindo a síntese e a comparação dos dados. O quadro facilitou uma observação interpretativa das informações a fim de responder à questão norteadora do estudo e colaborar com o resultado final da pesquisa.

Por tratar-se de um estudo de revisão de literatura que faz uso de dados de domínios públicos, não foi necessário submeter a pesquisa ao Comitê de Ética em Pesquisa (CEP), porém o estudo respeitou todos os aspectos éticos, assegurando a autenticidade de ideias, conceitos e definições aos autores pesquisados. 


\section{RESULTADOS}

Inicialmente, utilizou-se a base de dados SCIELO para a coleta dos artigos, sendo utilizado os descritores banho e recém-nascido, encontrando 12 artigos. Em seguida aplicou-se o critério de inclusão de publicação nos últimos cinco anos e texto completo, selecionando-se 6 artigos. Após foram excluídos os artigos de revisão de literatura, restando 4 artigos, os quais foram analisados através da leitura na íntegra e extração das informações para preenchimento do instrumento de coleta, confirmando assim a pertinência dos estudos à resposta do problema de pesquisa, sendo que 2 fugiram da temática, restando ao final 2 artigos selecionados para a revisão.

$\mathrm{Na}$ base de dados MEDLINE, utilizou-se os mesmos descritores, realizando o cruzamento deles e encontrando 771 artigos. Após aplicar o critério de inclusão de publicação nos últimos cinco anos, texto completo e assunto principal "banho", foram identificados 31 artigos. Em seguida foram excluídos artigos de revisão de literatura, restando 27 artigos. Estes foram lidos na íntegra, sendo que 23 fugiram da temática. Sendo assim, 4 artigos foram selecionado nesta base de dados.

No total foram localizados 783 artigos, sendo 777 reprovados após aplicação dos critérios de inclusão e exclusão, bem como da leitura e análise dos textos na íntegra. Portanto, foram elencados como relevantes para serem analisados na presente revisão 6 artigos (Figura 1).

Figura 1 - Fluxograma de seleção dos artigos para Revisão Integrativa.

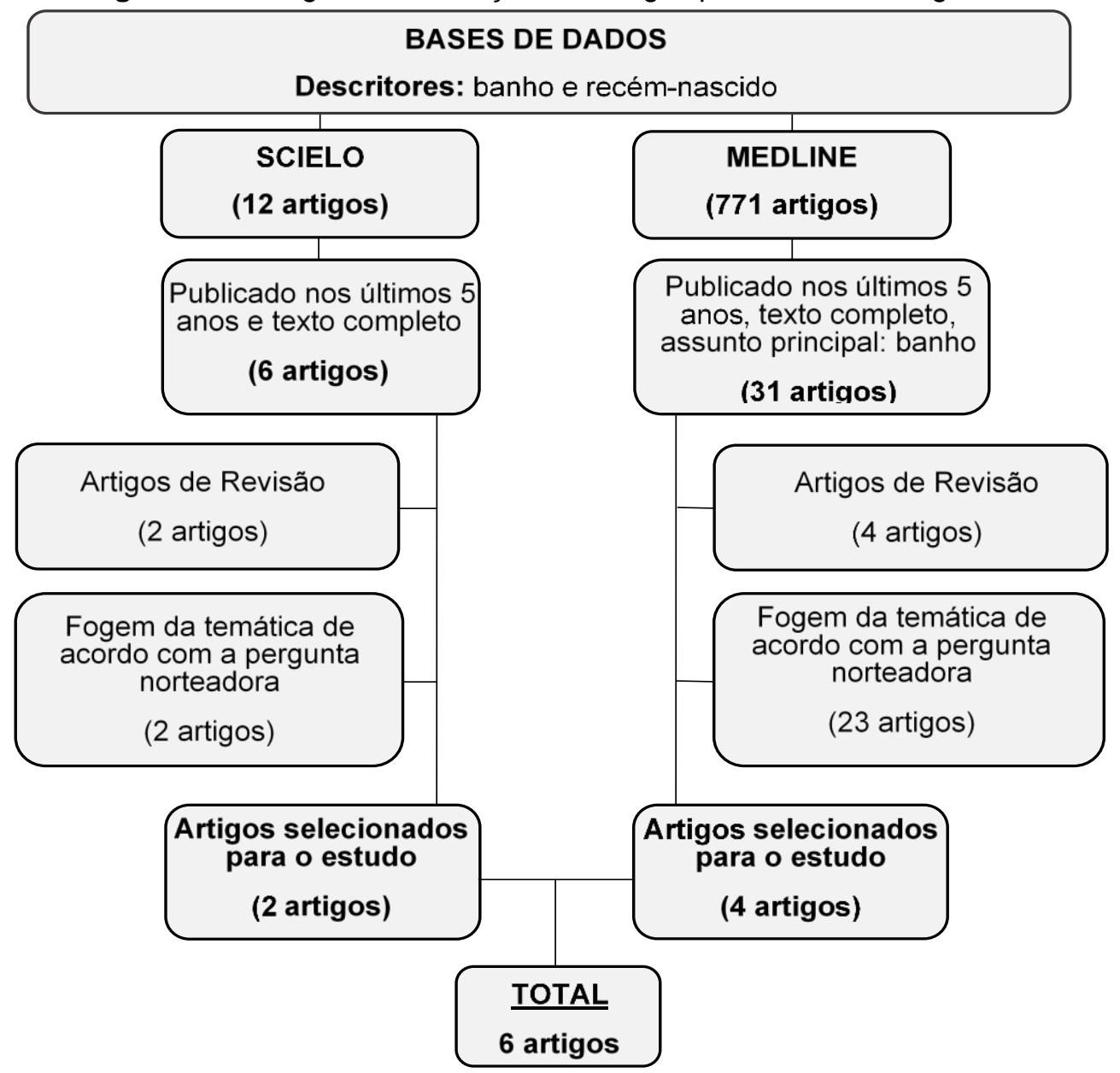

Fonte: Cantoni TS e Molin RSD, 2020.

Nesta revisão integrativa, foram analisados 6 artigos científicos que atenderam aos critérios de inclusão previamente estabelecidos. Os seguintes dados apresentam o sumário das características dos estudos incluídos e seus principais resultados (Quadro 1). 
Quadro 1 - Apresentação da síntese das características dos estudos incluídos na presente Revisão Integrativa.

\begin{tabular}{|c|c|c|c|c|c|c|}
\hline $\begin{array}{c}\text { Base de } \\
\text { dados }\end{array}$ & Título & Autores & $\begin{array}{c}\text { Periódico } \\
\text { (nome, local) }\end{array}$ & Objetivo & Resultados & Conclusões \\
\hline SCIELO & $\begin{array}{l}\text { Intervenção de } \\
\text { enfermagem- } \\
\text { primeiro banho do } \\
\text { recém-nascido: } \\
\text { estudo randomizado } \\
\text { sobre o } \\
\text { comportamento } \\
\text { neonatal }\end{array}$ & $\begin{array}{l}\text { LIMA RO, et } \\
\text { al., } 2020 .\end{array}$ & $\begin{array}{l}\text { Acta Paulista de } \\
\text { Enfermagem, } \\
\text { São Paulo, } \\
\text { Brasil. }\end{array}$ & $\begin{array}{c}\text { Avaliar os efeitos da } \\
\text { intervenção } \\
\text { Enfermagem- primeiro } \\
\text { banho sobre o choro e o } \\
\text { sono do recém- nascido. }\end{array}$ & $\begin{array}{l}\text { Os recém-nascidos do grupo intervenção do } \\
\text { estudo dormiram cerca de } 180 \text { minutos, não } \\
\text { apresentaram choro durante o experimento, e a } \\
\text { avaliação da escala de dor neonatal foi menor. }\end{array}$ & $\begin{array}{l}\text { A intervenção de enfermagem- } \\
\text { primeiro banho pode apresentar } \\
\text { melhora no estado comportamental } \\
\text { dos recém- nascidos. }\end{array}$ \\
\hline SCIELO & $\begin{array}{l}\text { Hipotermia e banho } \\
\text { do recém-nascido } \\
\text { nas primeiras horas } \\
\text { de vida }\end{array}$ & $\begin{array}{l}\text { RUSCHEL } \\
\text { LM, et al., } \\
2018 .\end{array}$ & $\begin{array}{l}\text { Revista Gaúcha } \\
\text { de Enfermagem, } \\
\text { Porto Alegre, } \\
\text { Brasil. }\end{array}$ & $\begin{array}{l}\text { Analisar a ocorrência de } \\
\text { hipotermia em recém- } \\
\text { nascidos antes e após o } \\
\text { banho nas primeiras } \\
\text { horas de vida. }\end{array}$ & $\begin{array}{c}\text { Foram incluídos } 149 \text { recém-nascidos no estudo, } \\
\text { evidenciando-se a prevalência de hipotermia } \\
\text { neonatal em } 40,3 \% \text { dos casos, tendo associação } \\
\text { com significância estatística }(p<0,001) \text { entre a } \\
\text { ocorrência de hipotermia neonatal em todos os } \\
\text { momentos de verificação de temperatura axilar. } \\
\text { Constatou-se correlação estatística significativa } \\
\text { entre as variáveis: temperatura da sala de parto e } \\
\text { a verificação da temperatura } 60 \text { minutos após o } \\
\text { banho }(p=0,032) \text {. }\end{array}$ & $\begin{array}{l}\text { Conclui-se que o primeiro banho } \\
\text { pode ser adiado para favorecer a } \\
\text { adaptação do neonato ao ambiente } \\
\text { extrauterino, prevenindo a } \\
\text { ocorrência de hipotermia neonatal. }\end{array}$ \\
\hline MEDLINE & $\begin{array}{c}\text { A troca de } \\
\text { procedimento de } \\
\text { banho de recém- } \\
\text { nascido pode alterar } \\
\text { a temperatura do } \\
\text { recém-nascido e a } \\
\text { amamentação } \\
\text { exclusiva? }\end{array}$ & $\begin{array}{l}\text { SUCHY C. } \\
\text { et al., } 2018 .\end{array}$ & $\begin{array}{l}\text { Neonatal } \\
\text { Network, } \\
\text { Califórnia, EUA. }\end{array}$ & $\begin{array}{l}\text { Avaliar os efeitos da } \\
\text { mudança do momento } \\
\text { do primeiro banho do } \\
\text { recém-nascidos na } \\
\text { temperatura infantil e } \\
\text { nas taxas de } \\
\text { amamentação exclusiva } \\
\text { antes da alta. }\end{array}$ & $\begin{array}{c}\text { De } 1.205 \text { recém-nascidos saudáveis de } 38 \\
\text { semanas, } 322 \text { nasceram antes da implementação } \\
\text { (Pré), } 486 \text { após (Post) e } 397 \text { durante a } \\
\text { manutenção (M). A adesão ao tempo de banho } \\
\text { aumentou e foi mantida: } 28 \text { por cento pré; } 83 \text { por } \\
\text { cento Post; } 85 \text { por cento M. Quase } 100 \text { por cento } \\
\text { dos recém-nascidos tiveram estabilidade nas } \\
\text { temperaturas. As taxas de exclusividade de } \\
\text { amamentação não mudaram ( } p \text {-valor } \geq 0,05) \text { : } \\
\text { banhos com menos de } 12 \text { horas: } 79 \text { por cento pré, } \\
74 \text { por cento pós e } 68 \text { por cento M; banhos } 12 \\
\text { horas: } 68 \text { por cento pré, } 71 \text { por cento pós e } 73 \text { por } \\
\text { cento M. }\end{array}$ & $\begin{array}{l}\text { A mudança do momento do banho } \\
\text { em recém-nascidos saudáveis } \\
\text { manteve a termorregulação e taxas } \\
\text { de aleitamento materno exclusivo. } \\
\text { Os enfermeiros mudaram a prática } \\
\text { rapidamente, mantendo a adesão ao } \\
\text { longo do tempo. }\end{array}$ \\
\hline
\end{tabular}




\begin{tabular}{|c|c|c|c|c|c|c|}
\hline $\begin{array}{c}\text { Base de } \\
\text { dados }\end{array}$ & Título & Autores & $\begin{array}{c}\text { Periódico } \\
\text { (nome, local) }\end{array}$ & Objetivo & Resultados & Conclusões \\
\hline MEDLINE & $\begin{array}{c}\text { Primeiro banho } \\
\text { tardio do recém- } \\
\text { nascido e taxas de } \\
\text { aleitamento materno } \\
\text { exclusivo }\end{array}$ & $\begin{array}{l}\text { TURNEY J. } \\
\text { et al., } 2019 .\end{array}$ & $\begin{array}{l}\text { Nurs Womens } \\
\text { Health, } \\
\text { Califórnia, EUA. }\end{array}$ & $\begin{array}{c}\text { Desenvolver um projeto } \\
\text { de prática baseada em } \\
\text { evidências para avaliar } \\
\text { o efeito de atrasar o } \\
\text { primeiro banho de um } \\
\text { recém-nascido em taxas } \\
\text { de amamentação na } \\
\text { alta. }\end{array}$ & $\begin{array}{c}\text { Observamos um aumento do tempo para o } \\
\text { primeiro banho de } 6,88 \text { horas para } 13,71 \text { horas ( } p \\
\leq 0,001 \text { ). O número de mulheres que optaram por } \\
\text { não dar banho em seus recém-nascidos durante a } \\
\text { internação hospital também aumentou cerca de } \\
\text { sete vezes, passando de } 0,16 \% \text { a } 1,1 \% \text {. A taxa de } \\
\text { aleitamento materno exclusivo na alta não mudou } \\
\text { significativamente após implementação ( } p \geq .05 \text { ), } \\
\text { independentemente de quando o primeiro banho } \\
\text { foi dado. }\end{array}$ & $\begin{array}{l}\text { O retardo do primeiro banho do } \\
\text { recém-nascido não foi associado a } \\
\text { um aumento nas taxas de } \\
\text { aleitamento materno exclusivo neste } \\
\text { projeto, mas há benefícios } \\
\text { potenciais, como menor risco de } \\
\text { hipotermia e hipoglicemia. Mais } \\
\text { pesquisas são necessárias para } \\
\text { determinar o efeito da hora do } \\
\text { banho. }\end{array}$ \\
\hline MEDLINE & $\begin{array}{l}\text { Atrasando o primeiro } \\
\text { banho do recém- } \\
\text { nascido e a } \\
\text { amamentação } \\
\text { exclusiva }\end{array}$ & $\begin{array}{l}\text { LONG K. et } \\
\text { al., } 2020 .\end{array}$ & $\begin{array}{l}\text { The American } \\
\text { Journal of } \\
\text { Maternal/ Child } \\
\text { Nursing, } \\
\text { California, EUA. }\end{array}$ & $\begin{array}{c}\text { Determinar se mudando } \\
\text { o momento do banho do } \\
\text { recém-nascido teria um } \\
\text { impacto na } \\
\text { amamentação exclusiva } \\
\text { durante a internação e } \\
\text { examinar a percepção } \\
\text { das enfermeiras os } \\
\text { benefícios e desafios } \\
\text { para tal mudança. }\end{array}$ & $\begin{array}{l}\text { Houve } 1.463 \text { mãe-bebê incluídos em três coortes } \\
\text { (A: } n=564 ; \text { B: } n=468 ; \mathrm{C}: \mathrm{n}=431) \text {. Não houve } \\
\text { significantes aumentos nas taxas de aleitamento } \\
\text { materno exclusivo (linha de base } 74,1 \%) \text { em } \\
\text { ambos os primeiros pós-implementadores coorte } \\
\text { de banho retardado }(70,1 \%, p=0,207) \text { e a } \\
\text { segunda coorte de "sustentabilidade" (79,4\%, } p= \\
0,060) \text {. Quinze das } 60 \text { enfermeiras completaram a } \\
\text { pesquisa, sendo uma taxa de resposta de } 25 \% \text {. } \\
\text { Temas gerados a partir de respostas da pesquisa } \\
\text { incluíam preocupações relacionadas a controle de } \\
\text { infecção, distribuição de trabalho, e benefícios da } \\
\text { prática (percepção de sucesso na amamentação, } \\
\text { diminuição da carga de trabalho). }\end{array}$ & $\begin{array}{l}\text { A taxa de amamentação exclusiva já } \\
\text { estava acima da média, assim como } \\
\text { a ser esperado em um hospital } \\
\text { designado Amigo da Criança e pode } \\
\text { ser uma razão pela qual não foi visto } \\
\text { uma mudança significativa na taxa } \\
\text { de amamentação. Ensaios } \\
\text { randomizados são necessários para } \\
\text { uma avaliação rigorosa do momento } \\
\text { do banho do recém-nascido e } \\
\text { possível relação com a } \\
\text { amamentação exclusiva no hospital } \\
\text { e após a alta. }\end{array}$ \\
\hline MEDLINE & $\begin{array}{l}\text { Implementação } \\
\text { baseada em } \\
\text { evidência do banho } \\
\text { de imersão } \\
\text { retardado realizado } \\
\text { pelos pais }\end{array}$ & $\begin{array}{l}\text { BROGAN J } \\
\text { e RAPKIN } \\
\text { G., } 2017 .\end{array}$ & $\begin{array}{l}\text { Nursing for } \\
\text { Women's Health, } \\
\text { California, EUA. }\end{array}$ & $\begin{array}{l}\text { Avaliar a eficácia da } \\
\text { mudança de prática do } \\
\text { primeiro banho sendo } \\
\text { realizada pelos pais } \\
\text { após } 24 \text { horas de vida } \\
\text { dos bebês. }\end{array}$ & $\begin{array}{l}\text { Uma análise dos dados de temperatura do recém- } \\
\text { nascido mostrou que os recém-nascidos que } \\
\text { receberam banhos de imersão retardados tiveram } \\
\text { menos probabilidade de ser hipotérmico do que } \\
\text { aqueles que receberam um banho de esponja logo } \\
\text { após o nascimento. Além disso, os pais relataram } \\
\text { que gostaram de dar o banho em seus recém- } \\
\text { nascidos e que se sentiam preparados para dar } \\
\text { banho em casa. }\end{array}$ & $\begin{array}{l}\text { O banho de imersão retardado é um } \\
\text { processo que pode ser } \\
\text { implementado em qualquer tipo de } \\
\text { estabelecimento médico, seja } \\
\text { grande ou pequeno. O custo é } \\
\text { mínimo e os benefícios de redução } \\
\text { do estresse causado pelo frio, maior } \\
\text { proteção da pele e maior satisfação } \\
\text { dos pais promovem resultados ideais } \\
\text { para os recém-nascidos. }\end{array}$ \\
\hline
\end{tabular}

Fonte: Cantoni TS e Molin RSD, 2020. 


\section{DISCUSSÃO}

Tendo em vista os estudos dos artigos selecionados nesta revisão integrativa, foram organizadas duas categorias analíticas: $O$ momento do primeiro banho no recém-nascido e suas implicações e a atuação do Profissional Enfermeiro nos benefícios do banho tardio.

\section{O momento do primeiro banho no recém-nascido e suas implicações}

De acordo com a recomendação da OMS (2017), o primeiro banho do recém-nascido deve ser atrasado em até 24 horas após o nascimento. O principal objetivo desta orientação está respaldado na proteção decorrente do vérnix caseoso, que está associado a função antimicrobiana, hidratação da pele, diminuição da descamação, redução do eritema tóxico neonatal e a prevenção da hipotermia neonatal, resultando na melhora da adaptação e da transição do neonato do meio intrauterino aquoso para o ambiente extrauterino seco.

Lima RO, et al. (2020) realizou um ensaio clínico randomizado controlado com 33 neonatos que tomaram seu primeiro banho após às 24 horas conforme preconizado pela OMS e identificou que a temperatura axilar após a intervenção foi mantida, prevenindo a hipotermia neonatal. Brogan J e Rapkin G (2017) também conduziram seu estudo possuindo como objetivo comparar o banho com esponja (aspersão) entre a segunda e quarta hora de vida e o banho de imersão após às 24 horas de vida (conforme preconizado), concluindo que, estes últimos apresentaram maior propensão a normotermia, além disso, identificou que o banho tardio possibilitou um maior tempo para a amamentação e envolvimento dos pais no cuidado com o recém-nascido.

Também seguindo as recomendações da OMS, um estudo realizado na Espanha, reafirmou que o banho deve ser adiado por no mínimo 24 horas, reiterando que este pode levar a hipotermia (sendo um fator desencadeante para a hipoglicemia neonatal), além de choro excessivo, aumento do consumo de oxigênio, angustia respiratória e alterações nos sinais vitais. Juntamente com esta recomendação, o estudo também enfatizou a não remoção precoce do vérnix caseoso em virtude dos benefícios e por não causar problemas de higiene (MONTEAGUDO B, et al., 2011).

Corroborando a esses achados, Ruschel LM, et al (2018), realizou um estudo transversal, comparando a ocorrência de hipotermia antes e após o banho nas primeiras horas de vida, sendo, o momento mais tardio, 4h. Dos 149 recém-nascidos analisados, 60 deles desenvolveram hipotermia, sendo que desses, 91,7\% haviam recebido o banho entre a primeira e segunda hora de vida, chegando a conclusão que mesmo utilizando-se de protocolos e critérios relacionados a temperatura da água, do ambiente e estado do RN o banho nas primeiras horas de vida pode interferir na termorregulação, devendo ser adiado por algumas horas.

Estudos buscaram relacionar o banho tardio com as taxas de amamentação. Dicioccio HC, et al. (2019), avaliou 996 recém-nascidos e evidenciou que o banho tardio (pelos menos $12 \mathrm{~h}$ ) foi associado a um aumento das taxas de aleitamento materno, além disso, identificou que o contato pele a pele diminuiu o estresse do RN durante o período inicial de adaptação. Suchy C, et al. (2018), Long K, et al. (2020) e Turney J, et al. (2018), com metodologias de estudo parecidas, não obtiveram o mesmo resultado quanto a amamentação, estes foram conduzidos em hospitais considerados Amigo da Criança e já possuíam taxas de amamentação exclusiva acima da média, diferente do estudo de Dicioccio, no entanto, também evidenciaram benefícios relacionados ao banho tardio superior às $12 \mathrm{~h}$ de vida, como o aumento do contato pele a pele, a estabilidade da temperatura corporal do recém-nascido, menores taxas de hipoglicemia, maior vínculo com a mãe, evitar o sono profundo do neonato que ocorria após o banho permitindo maior tempo para amamentação e melhora da pega, percepção da importância de manter o vérnix caseoso e menos estresse ao RN por não ser mais "esfregado" para remoção do mesmo.

O Ministério da Saúde preconiza o banho humanizado como sendo o mais indicado na assistência do RN, principalmente na modalidade de imersão, para auxiliar na termorregulação, de modo a evitar o estresse, a desorganização motora, o gasto energético e proporcionar relaxamento e prazer ao RN (BRASIL, 2013). Ruschel LM, et al (2018), Lima RO, et al. (2020), Suchy C, et al. (2018), Brogan J e Rapkin G (2017) utilizaram a modalidade de banho de imersão em seus estudos, atribuindo a conforto, menor perda de calor e maior tranquilidade do $\mathrm{RN}$ durante o procedimento. 
Pode-se observar a partir dos resultados, que os principais benefícios elencados pelos autores são relacionados ao efeito potencializador do primeiro banho tardio na saúde do recém-nascido, ou seja, quando associado a outros fatores como: temperatura da sala de parto/maternidade, iniciativas para promoção de amamentação, políticas humanizadas e educação em saúde (RUSCHEL LM, et al., 2018; LIMA RO, et al., 2020; LONG K, et al., 2020).

Como benefício "exclusivo" associado ao banho tardio, encontrou-se maior tempo de contato pele a pele, maior vínculo materno e participação dos pais no processo. Brogan J e Rapkin G (2017) trazem que a realização do banho após às 24 horas de vida, proporcionou a possibilidade do mesmo ser executado pelos próprios pais e aumentado a satisfação destes, os preparando para ida para casa com mais segurança. Suchy C, et al (2018), traz que ao esperarem no mínimo 12 horas após o nascimento permitiu-lhes obter mais relacionamento com os pais, os quais pareciam mais à vontade e receptivos à educação e ansiosos para participar do banho.

Cabe ressaltar, que dos estudos selecionados apenas os realizados por Lima RO, et al. (2020) e Brogan $\mathrm{J}$ e Rapkin G (2017) avaliaram o banho tardio considerando no minímo 24 horas de vida do RN e ambos obtiveram maiores resultados positivos quando comparados aos demais, principalmente relacionado a termorregulação.

\section{Atuação do Profissional Enfermeiro nos benefícios do banho tardio}

$O$ atendimento ao recém-nascido consiste na assistência por profissionais capacitados, dentre estes, destaca-se o profissional Enfermeiro, sendo preferencialmente especialista em obstetrícia ou cuidado neonatal. Um dos objetivos dessa assistência, é garantir que o RN seja mantido junto à sua mãe, sob supervisão e cuidados da equipe de enfermagem, possuindo o papel de minimizar o estresse das primeiras horas de vida do RN, acarretando em melhor resposta adaptativa, diminuição da dor, organização e padrão de sono e o mais importante, vínculo com a família. (BRASIL, 2014; BRASIL, 2017; PALHARES YLML, et al., 2016).

Neste contexto, Lima RO, et al. (2020), buscou identificar o impacto da intervenção enfermagem-primeiro banho sobre o choro e o sono do recém-nascido, concluindo que estes choraram menos, ou seja, apresentaram um comportamento de conforto, além de ostentar um período de sono mais longo e exibir mediante a Escala de Dor em Neonatos (NIPS), menor sinal de dor e manter a temperatura corporal estável. A intervenção se mostrou importante para o $\mathrm{RN}$, visto que, o sono constitui um fator de equilíbrio de recuperação de energia, e a presença do desconforto da dor, constitui um estressor e pode acarretar alterações fisiológicas nocivas ao RN (LIMA RO, et al., 2020).

O primeiro banho do recém-nascido é uma prática que deve ser realizada de maneira cuidadosa, pois o bebê ainda está sofrendo adaptações à vida extrauterina e sua pele é vulnerável a lesões, a equipe de enfermagem deve estar apta a realizar o procedimento no momento adequado, utilizando-se de agentes certos, promovendo conforto e menor estresse ao $\mathrm{RN}$, proporcionando um momento calmo e tranquilizante. Além disso, a enfermagem também deve estar atenta a todos os cuidados, como por exemplo: o preparo e condições do ambiente físico, a temperatura do ambiente e da água utilizada para o banho, os materiais e recursos adequados, às condições físicas, a avaliação do bebê, medidas de conforto durante a imersão na água e as condições da mãe (BARCELOS AA e ZANI AV, 2017; SCHNEIDER AS, 2015).

Nos demais estudos elencados nesta revisão, utilizou-se o profissional enfermeiro também como agente de mudança da prática do primeiro banho imediato para o tardio, possuindo o papel de educar as mulheres sobre os benefícios de atrasar o primeiro banho. No estudo de Turney J, et al. (2018), a atuação deste profissional resultou em uma significativa mudança de prática aumentando o tempo médio do primeiro banho do recém-nascido de 6,88 horas para 13,71 horas após o nascimento, além de observarem um aumento na taxa de mulheres que optaram por não realizarem o banho em seus recém-nascidos durante a internação de $0,16 \%$ para $1,1 \%$.

O enfermeiro também possui papel fundamental nos cuidados que a família vai desenvolver com o recémnascido, por meio da educação em saúde (BRASIL, 2014). No estudo desenvolvido por Brogan J e Rapkin G (2017), o enfermeiro era responsável por instruir os pais sobre como dar banho em seus recém-nascidos e 
eram encorajados a realizá-lo, isso se deu a partir da percepção de que a hora do banho é um momento de grande oportunidade para os profissionais ensinarem a partir de evidências científicas, a forma adequada aos pais de como cuidar da pele do seu recém-nascido, além de permitir a aprendizagem prática.

Por fim, os resultados demonstram ainda, a capacidade da enfermagem de implementar a prática da realização tardia do primeiro banho e mantê-la ao longo do tempo. O custo para efetivação é mínimo e seus maiores benefícios encontraram-se na redução do estresse causado pelo frio, maior proteção da pele e aumento da satisfação dos pais promovendo resultados imediatos no recém-nascido (BROGAN J e RAPKIN G, 2017; LONG K, et al., 2020).

\section{CONSIDERAÇÕES FINAIS}

O banho tardio preferencialmente após às 24 horas de vida está diretamente relacionado a prevenção da hipotermia neonatal e a não remoção precoce do vérnix caseoso. Possui efeito potencializador no sucesso da amamentação e principalmente na promoção de um maior tempo de contato pele a pele, proporcionando vínculo materno e a inserção dos pais nos cuidados com o recém-nascido. Nesse contexto, o enfermeiro está atrelado a educação em saúde e a diminuição do estresse do $\mathrm{RN}$ no seu primeiro banho, possuindo capacidade de implementar práticas baseadas em evidências sustentadas ao longo do tempo. Recomenda-se que novas pesquisas sejam realizadas para esclarecer o impacto do banho nas primeiras horas de vida e o banho tardio superior a 24 horas no processo de adaptação fisiológica do RN.

\section{REFERÊNCIAS}

1. BARCELLOS AA, ZANI AV. O Primeiro Banho No Prematuro Hospitalizado: Revisão Integrativa. Brazilian Journal of Surgery and Clinical Research - BJSCR, 2017; 20(1): 128-133.

2. BRASIL. Ministério da Saúde. Atenção Humanizada ao Recém-Nascido de Baixo Peso Método Canguru: Manual Técnico. 2013. Disponível em: https://bvsms.saude.gov.br/bvs/publicacoes/atencao_humanizada_metodo_canguru_manual_3ed.pdf. Acesso em: 11 out. 2020.

3. BRASIL. Ministério da Saúde. Atenção à saúde do recém-nascido: guia para os profissionais de saúde. 2014. Disponível em: https://bvsms.saude.gov.br/bvs/publicacoes/atencao_saude_recem_nascido_v1.pdf. Acesso em: 11 out. 2020.

4. BRASIL. Ministério da Saúde. Atenção Humanizada ao Recém-Nascido: Manual Técnico. 2017. Disponível em: https://bvsms.saude.gov.br/bvs/publicacoes/atencao_humanizada_metodo_canguru_manual_3ed.pdf>. Acesso em $11 \mathrm{de}$ out. 2020

5. BROGAN J; RAPKIN, G. Implementing evidence-based neonatal skin care with parent-performed, delayed immersion baths. Nursing for Women's Health, 2017; $21(6):$ 442-450.

6. CARVALHÊDO DS., et al.. As vivências e os significados dos primeiro banho dado pela puérpera em seu filho recém-nascido. Enfermería Global, 2010; 19.

7. CUNHA AL. Práticas Culturais do Primeiro Banho do Recém-Nascido em Alojamento Conjunto: contribuições da enfermagem neonatal. Dissertação (Mestrado em Enfermagem) Escola de Enfermagem Anna Nery. Universidade Federal do Rio de Janeiro, Rio de Janeiro, 2013; 104 p.

8. COOPER HM. Scientific guidelines for conducting integrative research reviews. Review of educational research, 1982; 52(2): 291-302.

9. FREITAS P, et al. Variações nos parâmetros fisiológicos e comportamentais de recém-nascidos pré- termo submetidos à higienização corporal: revisão sistemática. Revista da Escola de Enfermagem da USP, 2014; 8(spe): 178-183.

10. LIMA RO, et al. Intervenção de enfermagem-primeiro banho do recém-nascido: estudo randomizado sobre o comportamento neonatal. Acta Paulista de Enfermagem, 2020; 33.

11. LONG K, et al. Delaying the First Newborn Bath and Exclusive Breastfeeding. The American Journal of Maternal/Child Nursing, 2020; 45(2): 110-115

12. MENDES KDS, et al.Revisão integrativa: método de pesquisa para a incorporação de evidências na saúde e na enfermagem. Texto \& Contexto-Enfermagem, 2008; 17(4): 758-764.

13. MONTEAGUDO B, et al. Influence of neonatal and maternal factors on the prevalence of vernix caseosa. Actas DermoSifiliográficas, 2011; 102(9): 726-729.

14. PALHARES YLML, et al. Conhecimento dos profissionais de enfermagem quanto à realização do banho no recém-nascido. Revista Enfermagem Atual InDerme, 2016; 78(16).

15. PRIORE MD. História das crianças no Brasil. $7^{\mathfrak{a}}$ edição, São Paulo: Contexto, 2010; 450p.

16. PUGLIESI VEM, et al. Efeitos do banho logo após o nascimento sobre as adaptações térmica e cardiorrespiratória do recémnascido a termo. Revista Paulista de Pediatria, 2009; 27(4): 410- 415, 2009.

17. RUSCHEL LM, et al. Hipotermia e banho do recém-nascido nas primeiras horas de vida. Revista Gaúcha de Enfermagem,

18. SCHNEIDER AS. Repercussões do Primeiro Banho na Pele Do Recém-Nascido: Uma Revisão Integrativa. Trabalho de Conclusão de Curso de Graduação em Enfermagem - Escola de Enfermagem. Universidade Federal do Rio Grande do Sul, Porto Alegre, 2015; 34p.

19. SILVA SCSPD, et al. Critérios clínicos e insumos utilizados no banho de recém-nascidos pré-termo de muito baixo peso. Enfermagem em foco, 2020; 11(2): 127-132.

20. SUCHY C, et al. Does changing newborn bath procedure alter newborn temperatures and exclusive breastfeeding?. Neonatal Network, 2018; 37(1): 4-10.

21. TURNEY J, et al. Delayed newborn first bath and exclusive breastfeeding rates. Nursing for women's health, $2019 ; 23$ (1): 31-37.

22. WHO. World Health Organization. WHO recommendations on newborn health: Guidelines approved by the WHO guidelines review committee. 2017. Disponível em: https://apps.who.int/iris/handle/10665/259269. Acesso em 17 nov. 2020. 